\title{
Experimental study of microwave properties of composites filled with Permalloy powder
}

\author{
Stanislav Y. Bobrovskii*, Victor A. Garanov, Andrei S. Naboko, Alexei V. Osipov and Konstantin N. Rozanov \\ Institute for Theoretical and Applied Electromagnetics RAS
}

\begin{abstract}
Frequency dependencies of permittivity and permeability of composites filled with Permalloy powder with various concentrations and shapes of particles have been measured and discussed. The powder particles of various shapes were obtained by mechanical milling of industrial Permalloy powder in organic media. Paraffin wax was used as a matrix for producing the composites. A shape data of the particles was obtained with an electron microscope. The permittivity and permeability were measured in the frequency range of 0.13 to $10 \mathrm{GHz}$. The shape of the particles changes from sphere to platelet with increase of milling time. The change leads to a shift of the ferromagnetic resonance (FMR) frequency and magnetic loss peak. It is shown that for larger particles, a decrease of the Q-factor of the ferromagnetic resonance is observed, which is explained by the strong influence of the skin effect. With increase of the concentration of the Permalloy particles in composite, the magnetic loss peak shifts to low frequencies. When the spherical particles are flattened, the resonance frequency shifts to high frequencies, which indicates that the shift to high frequencies due to the decrease of the skin effect and the change in the magnetic structure is stronger than the shift to the low frequencies by changing the shape and reducing the demagnetization.
\end{abstract}

\section{Introduction}

The frequency dependencies of microwave permeability have a complex shape in many materials. Analysis of such dependencies is a challenging problem [1-4], that allows, in particular, obtaining estimates of the largest feasible values of microwave permeability. In this regard, the relations between the parameters of the magnetic loss peak are important, because such relations allows the number of independent variables used in describing the magnetic spectra to be reduced.

In the simplest case, the dependence of the complex permeability $\mu=\mu^{\prime}+i \mu^{\prime \prime}$ on frequency $f$ obeys the Lorentzian dispersion law:

$$
\mu(f)=1+\frac{\mu_{s}-1}{1-\frac{\mathrm{i} f}{f_{1}}-\left(\frac{f}{f_{0}}\right)^{2}},
$$

where $\mu_{s}$ is the static permeability, $f_{0}$ is the resonant frequency, $f_{1}$ is the relaxation frequency. According to (1), the real permeability is close to $\mu_{s}$ at frequencies below $f_{0}$, and falls sharply at higher frequencies. In this way, $f_{0}$ can be considered as the cut-off frequency of the permeability. For many magnetic materials $\mu_{s}$ and $f_{0}$ are related by Snoek's law [5]:

$$
\left(\mu_{s}-1\right) f_{0}=(2 / 3) \gamma 4 \pi M_{0},
$$

where $4 \pi M_{0}$ is the saturation magnetization of the material, $\gamma \approx 3 \mathrm{GHz} / \mathrm{kOe}$. Therefore, the higher the static permeability of the material, the lower frequencies where dynamic magnetic properties starts to disappear. A measure of the high-frequency magnetic properties of a material is the quantity on the right-hand side of (2), is Snoek's parameter $S$.

Snoek's law relates the parameters of the permeability spectrum - the amplitude and position of the absorption peak, which depend substantially on the crystalline and magnetic structure of the material, with the saturation magnetization determined solely by the composition. Snoek's law follows from the LandauLifshitz equation that describes the ferromagnetic resonance in a single-domain spherical particle.

If the magnetic particles are in shape of thin plates and their magnetic moment is in the plane of the plate, then[6]:

$$
\left(\mu_{s}-1\right) f_{0}^{2}=\left(\frac{1}{2}\right)\left(\gamma 4 \pi M_{0}\right)^{2},
$$

where the factor $1 / 2$ corresponds to the uniform orientation of the magnetic moments in the plane.

It is seen from (3) that thin ferromagnetic films can possess the highest values of microwave permeability of all magnetic materials. The right-hand side of (3) is a measure of the high-frequency properties of materials based on thin films and is called Acher's parameter $A$.

Composites with conducting particles are of interest for a number of practical applications. This study is devoted to the composites filled with Permalloy powder. The effect of concentration, size and shape of inclusions on the properties of composites is investigated.

\footnotetext{
* Corresponding author: sbobrowskiy@yandex.ru
} 

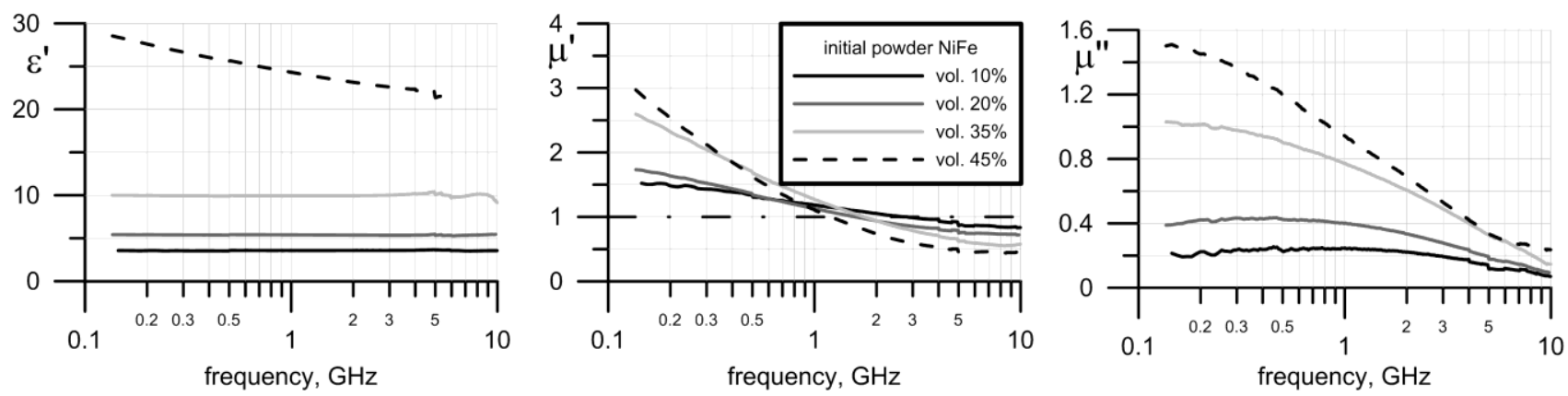

Fig. 1. The measured frequency dependencies of the permittivity and permeability of the composites with initial Permalloy powder with various volume concentration.

\section{Experimental details}

The shape of the particles of the powders under study was transformed by high-energy mechanical milling of the Permalloy powder. The initial powder consisted of spherical particles and was separated into four fractions by particle size: less than $40 \mu \mathrm{m}$, from 40 to $63 \mu \mathrm{m}, 63$ to 90

$\mu \mathrm{m}$ and above $90 \mu \mathrm{m}$. Milling time varied from 20 to 320 seconds. Images of powders were obtained using an electron microscope.

Samples for a measurement were prepared by manual stirring the necessary amount of Permalloy powder and molten paraffin wax to form a uniform distribution of inclusions in the composite. The permittivity $\varepsilon$ of paraffin wax is 2.25 . Then the mixture was placed in a mold to give the sample a shape corresponding to the measuring cell. The thickness of the samples was usually chosen in the range from 1 to $5 \mathrm{~mm}$.

Measurements of material parameters (permittivity and permeability) were performed using a standard coaxial line of $7 / 3 \mathrm{~mm}$ in the frequency range of 0.13 to $10 \mathrm{GHz}$ and HP8720A vector analyzer. The permittivity and permeability were calculated from the measures of the reflection coefficients, with the short-circuited and opencircuited sample [7].

\section{Results}

Figure 1 shows the measured frequency dependencies of material parameters of composites for different volume concentrations of initial Permalloy powder. The imaginary permittivity is close to zero and is of no interest. With an increase of the concentration of inclusions, the frequency of the resonance and the magnetic loss peak shifts to low frequencies, which is in agreement with the effective medium theory [8].

The permittivity of the composites under study does not depend on the frequency in the measured frequency range, and dielectric loss is small. The values of the real permeability at high frequencies drop below unity up to the level corresponding to the volume concentration of inclusions, which indicates a strong influence of the skin effect [9]. The magnetic loss peak is half the amplitude of the ferromagnetic resonance, so as the values of $\mu_{s}-1$. The shift to low frequencies with increasing concentration can be associated with proximity to the percolation threshold. The ratio between the absorption maximum and the amplitude indicates a high damping of the resonance: the shape of the curve corresponds to the Debye formula, so the spectrum is very spread.

In Fig. 2, the measured frequency dependencies of the material parameters of composites with particles of different sizes are shown.

The permittivity of all powders is almost the same, which indicates the same shape of the particles. The permeability shifts to low frequencies, both in terms of the resonance frequency $\left(\mu^{\prime}=1\right)$ and of the frequency of the maximum loss, which corresponds to the ferromagnetic resonance theory. For particles larger than $90 \mu \mathrm{m}$, a decrease in the amplitude without a shift in frequency is
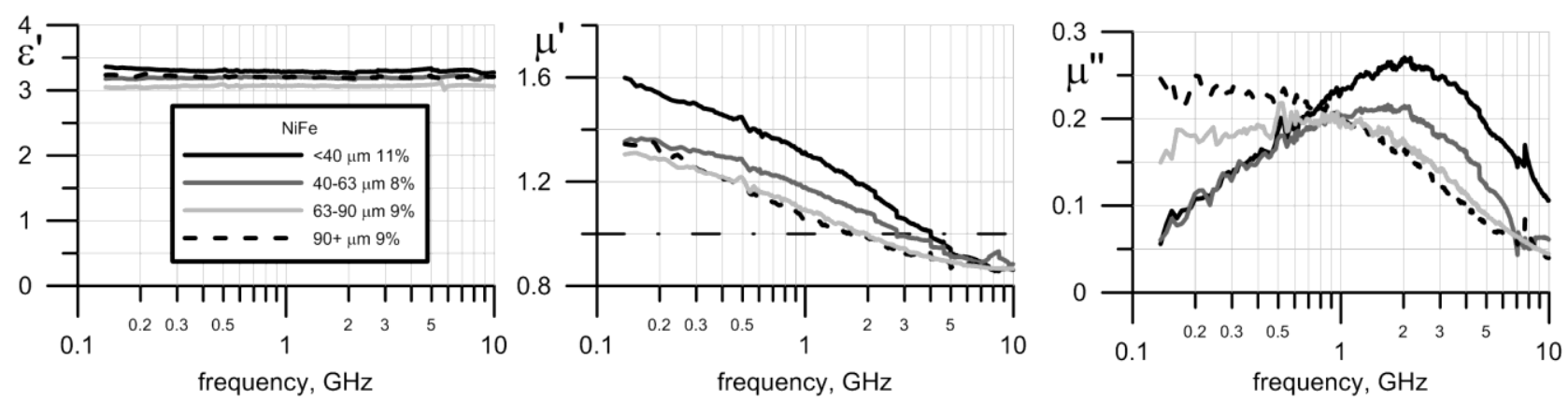

Fig. 2. The measured frequency dependencies of the composites with initial Permalloy powder with various size of the particles, volume concentration $\approx 10 \%$ 


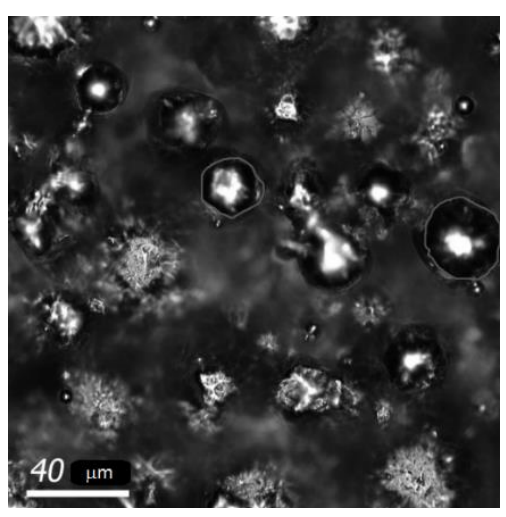

(a)

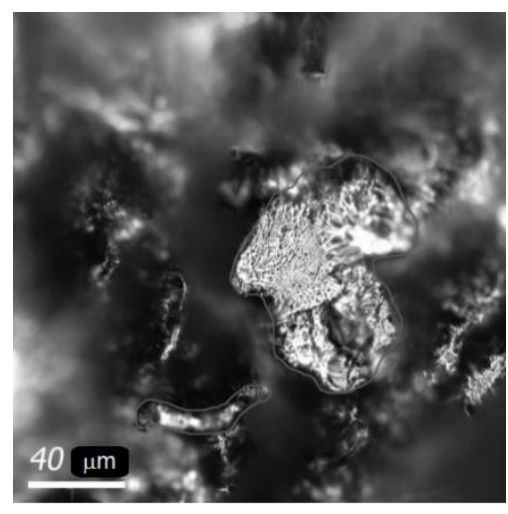

(b)

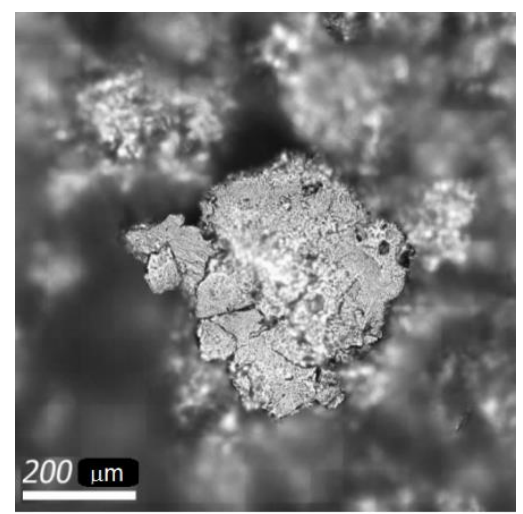

(c)

Fig.3. Optical representation of the initial powder with particle size up to $40 \mu \mathrm{m}$ (a), after $80 \mathrm{~s}$ milling (b), after $160 \mathrm{~s}$ milling (c)

observed, as compared with $63-90 \mu \mathrm{m}$ particles. The frequency of ferromagnetic resonance for the smallest particles is $4 \mathrm{GHz}$ or higher. Since for conductive particles the permittivity is determined only by the shape, then the equality of $\varepsilon$ implies the similarity of the shape. As is shown below, the powder particles of all fractions are in the form of spheres. Consequently, the cause of the change in permeability is the influence of size, i.e. skin effect. This is confirmed by a decrease in the Q-factor with increasing size, as can be seen from the broadening of the magnetic loss peak.

A method to avoid the influence of the skin effect is to change the shape of the particles by flattening. This should reduce the skin effect, which is determined by the least particle size, and the ferromagnetic resonance should shift to the lower frequencies by reducing the effective demagnetization factor.

To determine the shape of the particles under study, an optical image of the powder of the fraction up to $40 \mu \mathrm{m}$ was obtained with a confocal microscope (Fig. 3a). The image was made with $\mathrm{x} 50$ lens. A sample of the powder consists of granules with a diameter of 10-30 $\mu \mathrm{m}$ (in the plane, i.e., in area). The variation in the focal length in a confocal microscope makes it possible to estimate the size of the particles in height, which is also 20-30 $\mu \mathrm{m}$. Consequently, the considered particles have a nearspherical shape; the aspect ratio is close to 1:1.

After milling for $t_{m}=80 \mathrm{~s}$ (Fig. 3b), the shape of the powder particles changed from spherical to platelet. The particle diameter in the plane is $50-80 \mu \mathrm{m}$, the thickness is $10 \mu \mathrm{m}$, while the particles have a slight bending in the plane. The aspect ratio is $1: 5-1: 8$, therefore the particles are flattening during milling. In Fig. $3 \mathrm{c}$ there is an optical image of the powder milled for $t_{m}=160 \mathrm{~s}$. After milling for 160 seconds, the particle thickness is less than $2-3 \mu \mathrm{m}$. The diameter of the particles in the plane has a range from 50 to $500 \mu \mathrm{m}$, and many smaller fragments.

Frequency dependencies of permittivity and permeability of composites of the Permalloy powder with the various milling time were measured. The particle size before milling were in range of 63 to $90 \mu \mathrm{m}$ with volume concentration of the inclusions $p=0.1$. The measured result is presented at the Fig. 4.

It is seen that the permittivity increases uniformly up to $\varepsilon=18$, then decreases at $t_{m}=320 \mathrm{~s}$. The permeability grows in amplitude up to $t_{m}=240 \mathrm{~s}$, the resonance frequency and the frequency of maximum loss slowly shifts to higher frequencies. At $t_{m}=320 \mathrm{~s}$ the amplitude decreases, the resonance frequency continues to increase, the magnetic loss peak shifts to high frequencies from 1 to $3 \mathrm{GHz}$ and decreases in amplitude. For milling time $t_{m}=240 \mathrm{~s}$, Acher's and Snoek's parameters are $A=0.6$ $\mathrm{GHz}^{2}, S=2.4 \mathrm{GHz}$, this indicates that the magnetic structure is in some intermediate state between Snoek's law (spherical particles) and Acher's law (planar particles with inplane magnetization).

The magnitude of the absorption maximum is half the amplitude, hence, from the point of view of the frequency mechanism, relaxation is observed. The change in $\varepsilon$ indicates that the particles are flattened up to $t_{m}=240 \mathrm{~s}$, then their milling occurs, so that a significant part of the powder is a number of small fragments. When the


Fig.4. The measured frequency dependencies of composites with Permalloy powder $63-90 \mu \mathrm{m}$, volume concentration $\approx 10 \%$, with various milling time $t_{m}$ 
spherical particles are flattened, the resonance frequency should shift to lower frequencies. The shift in the opposite direction can be caused both by a decrease in skin effect and by a change in the magnetic structure of the particles. The decrease in skinning is confirmed by the increase of the Q-factor, based on the values of Acher's and Snoek's parameters. It may be concluded that the magnetic structure of the particles is predominantly closer to bulk polycrystalline that obeys Snoek's law.

\section{Conclusions}

The frequency dependencies of permittivity and permeability of composites for fractions of different sizes were measured. It is shown that for larger particles, it is a decrease of the Q-factor of the ferromagnetic resonance, which is explained by the strong influence of the skin effect. Frequency dependencies of permittivity and permeability were measured for different milling time. It is shown that as the milling time increases, the amplitude of the magnetic permeability increases. The maximum of magnetic loss and the frequency of the ferromagnetic resonance shifts to high frequencies. It is shown that due to milling, the shape of the particles changes from spherical to platelet, and the calculated values of Acher's and Snoek's parameters indicate that the magnetic structure of the resulting particles is closer to a bulk polycrystalline that satisfies Snoek's law. When the spherical particles are flattened, the resonance frequency shifts to high frequencies, which indicates that the shift to high frequencies due to the decrease of the skin effect and the change in the magnetic structure is stronger than the shift to low frequencies by changing the shape and reducing the demagnetization.

The authors acknowledge financial support from Russian Science Foundation (RSF), according to agreement no. 16-1910490.

\section{References}

1. S.S.Maklakov, A.N.Lagarkov, S.A. Maklakov, Y.A. Adamovich, D.A. Petrov, K.N. Rozanov, I.A. Ryzhikov, A.Y. Zarubina, K.V. Pokholok, D.S. Filimonov, J Alloys Compounds, 706 (2017) 267273.

2. S.F. Lomayeva, A.V. Syugaev, A.N. Maratkanova, A.A. Shakov, K.N. Rozanov, D.A. Petrov, C.A. Stergiou, J. Alloys Compounds, 721 (2017) 18-27.

3. S.N. Starostenko, K.N. Rozanov, A.O. Shiryaev, A.N. Shalygin, and A.N. Lagarkov, J. Appl. Phys. 121, 245107 (2017).

4. X.G. Huang, J. Zhang, W.F. Rao, T.Y. Sang, B.Song, C.P. Wong, J. Alloys Compounds, 662, 409 (2016)

5. J.L. Snoek, Physica, 14, 207 (1948)

6. O. Acher, P.-M. Jacquart, J.-M. Fontaine, Ph. Baclet, and G. Perrin, IEEE Trans Magn., 30, 4533 (1994)

7. K.N. Rozanov, N.A. Simonov, A.V. Osipov, J. Commun. Technol. Electron., 47, 229 (2002)
8. A.N.Lagarkov, K.N.Rozanov, J. Magn. Magn. Mater., 321, 2082 (2009)

9. L. Lewin, Theory of Waveguides, (John Wiley \& Sons, Canada, 1975) 